\title{
Application of control volume numerical method in thermographic analysis of relative material loss
}

by S. Švaić and I. Boras

Faculty of mechanical engineering and naval architecture

University of Zagreb, Croatia

\section{Abstract}

Application of IR thermography for revealing and estimation of corrosion intensity seems to have a good perspective as a non destructive method. Beside the limitations which are the results of IR camera itself and thermal properties of the material detected, when combined with adequate numerical method the acceptable results can be obtained.

The paper presents the comparison of the results obtained by simulation by means of software developed by authors and the results obtained by thermographic measurement presented by Marinetti, Bison and Grinzato in reference [1].

\section{Introduction}

The model for the simulation of corrosion defects was a steel plate with dimensions $120 \times 80 \times 3 \mathrm{~mm}$, as shown in Figure 1. The corroded areas are represented by six cylindrical recesses of $10 \mathrm{~mm}$ diameter. The depth of each defect represents the particular loss of material caused by corrosion. For the numerical part of the investigation, the corrosion is defined as a reduction of material thickness, neglecting any changes in thermal properties of the material that may occur due to chemical reactions involved in the corrosion process.

The smooth front surface of the model was stimulated at the beginning of the simulation with a heat flux having the total energy amount of $78 \mathrm{~J}$ in a $5 \mathrm{~ms}$ time interval. The model was made of steel with known thermal properties: thermal conductivity $\lambda=62 \mathrm{~W} /(\mathrm{mK})$, thermal diffusivity $a=1,65 \cdot 10^{-5} \mathrm{~m}^{2} / \mathrm{s}$.

It is assumed that the thermal stimulation is uniform along the sample surface.

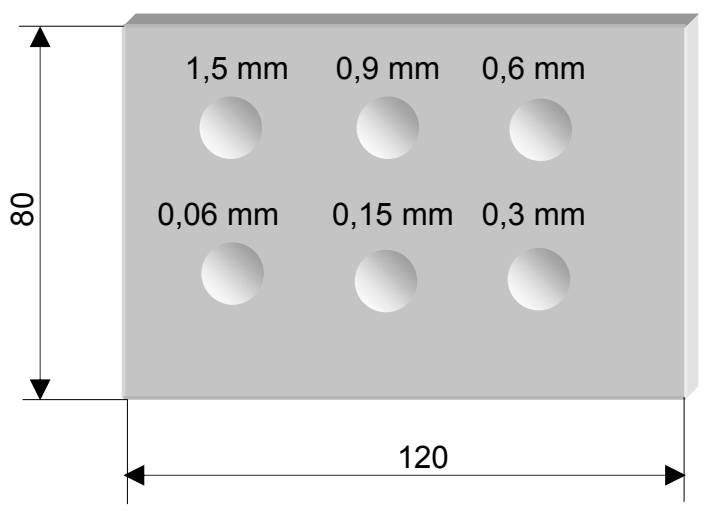

Fig. 1. The sample 


\section{Results of numerical simulation}

The problem was solved numerically by using the control volume method. For solving the system of algebraic equations, the Gauss-Seidel procedure was used. The total number of control volumes was 29 120. The control volume mesh was adapted to the observed problem. The mesh was condensed in the areas where steeper temperature gradients were expected.

The time step was also adapted to the stability and accuracy requirements of the discretized equation, so $\Delta t=0,001 \mathrm{~s}$ was adopted for defined model. The initial condition of the simulation presumed a model of uniform temperature.

The investigations at model were aimed to show the possibility of detection of corrosion at thin metal plates. In this paper the results of the numerical simulation by the authors are shown, and compared to data obtained experimentally by Marinetti, Bison and Grinzato [4].

The temperature distributions at the plate surface for different time increments are shown in Figure 2.

The comparison of numerical and experimental results, as shown in literature [4] using the temperature distribution along the white dotted line in the thermogram for the time increment $280 \mathrm{~ms}$ in Figure 3. It displays very good accordance.
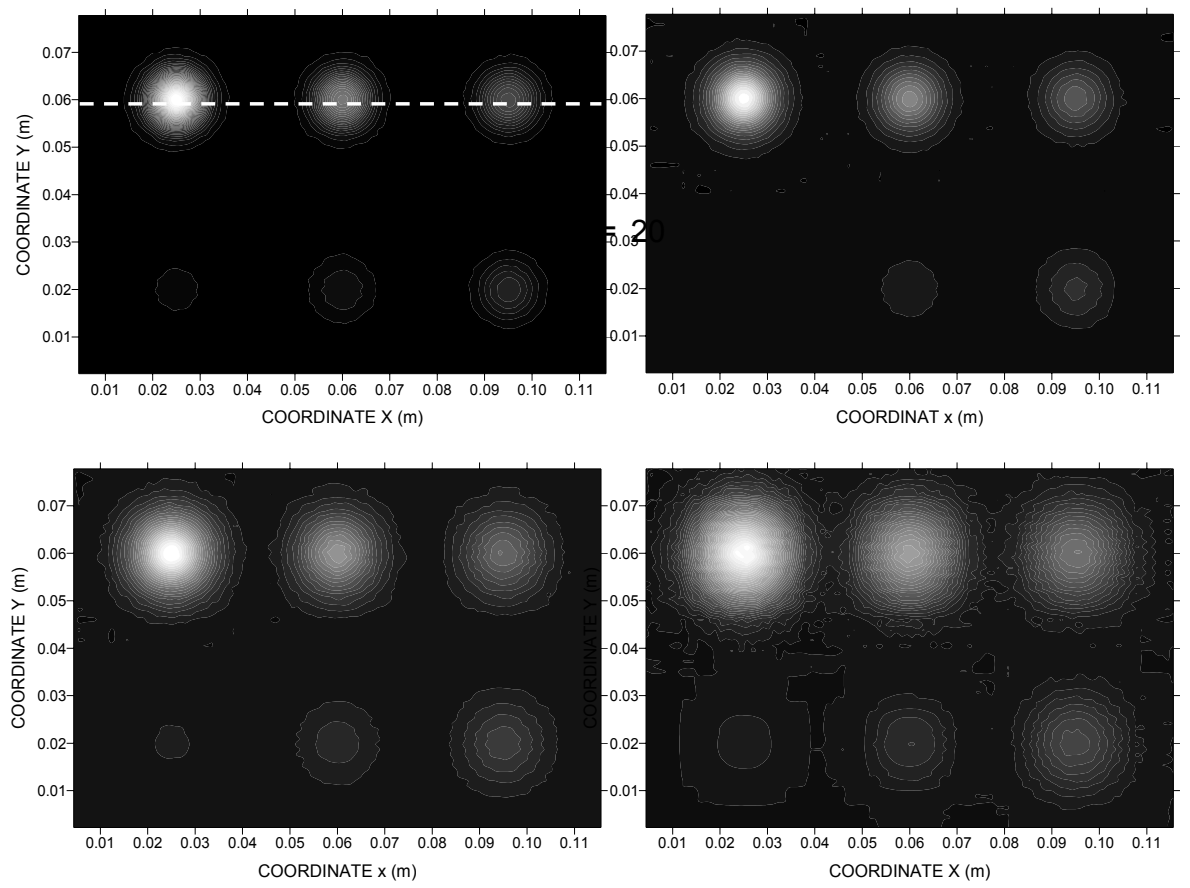

Fig. 2. Numerically obtained temperature distributions at the front plate surface at $280 \mathrm{~ms}, 400 \mathrm{~ms}, 600 \mathrm{~ms}$ and $1000 \mathrm{~ms}$ time increments 
http://dx.doi.org/10.21611/qirt.2006.063

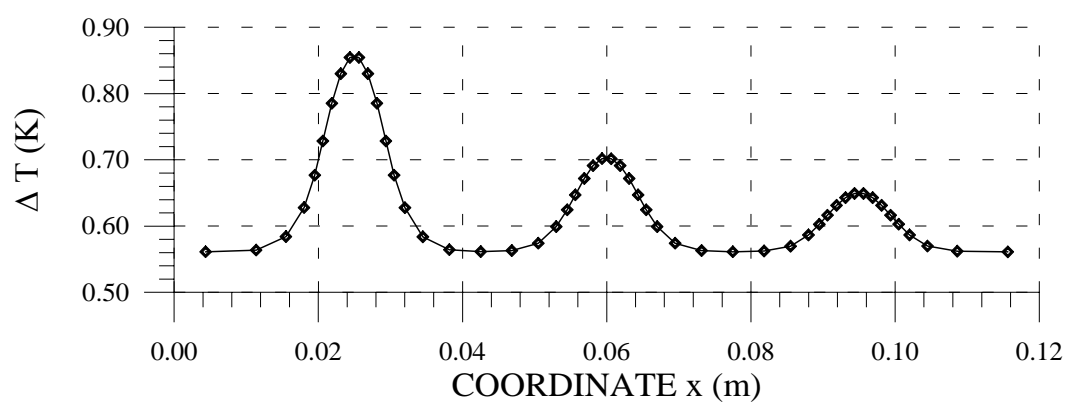

Fig. 3. The temperature distribution along the dotted line from fig. 2

The corrosion degree estimation may be performed by using one of the inverse methods. The simplest algorithm connects the relative material loss and the instantaneous contrast through the equation:

$$
\frac{\Delta L}{L}=\frac{C\left(x, y, \tau_{\infty}\right)}{1+C\left(x, y, \tau_{\infty}\right)}
$$

The time interval during which the temperature response at the material surface is observed is most frequently expressed nondimensionally, i.e. using the Fourier number:

$$
F O=\frac{a \cdot \tau}{L^{2}}
$$

where $a$ - thermal diffusivity of the material, $\left(\mathrm{m}^{2} / \mathrm{s}\right), \tau$ - time interval, (s), and $L$ - thickness of the plate, $(m)$.

For large defects the heat transport may be reduced to a one-dimensional problem. The optimal inspection time is in the range Fo $\approx 0,6 \div 2,0$, and equation (1) is recommended. The characterization of smaller defects of complex shape is recommended in the interval $\mathrm{Fo} \approx 0,3 \div 0,6[5]$.

Due to the three-dimensional heat diffusion, in this case the detection is more difficult, so it is also recommended to use the temperature derivation by time, according to the equation:

$$
M(x, y)=\frac{T\left(x, y, \tau_{1}\right)-T\left(x, y, \tau_{2}\right)}{\left(\tau_{1}-\tau_{2}\right)}
$$

In any case, the shape of the corroded area is more precisely indicated in a shorter inspection time interval, but with somewhat lower amplitude [5].

Figure 4 displays the temporal distribution of values according to equation (1). In contrast to the data from [4], where the analysis was done for Fo $=0,68$, the numerical simulation shows that the best results are obtained by inspection during the time of maximal temperature contrast [3]. For all the defects this was in the range $\mathrm{Fo} \approx 0,21 \div 0,32$. 
It is also important to select the referent point $T_{\text {nd }}$ sufficiently far from the defect itself [6].

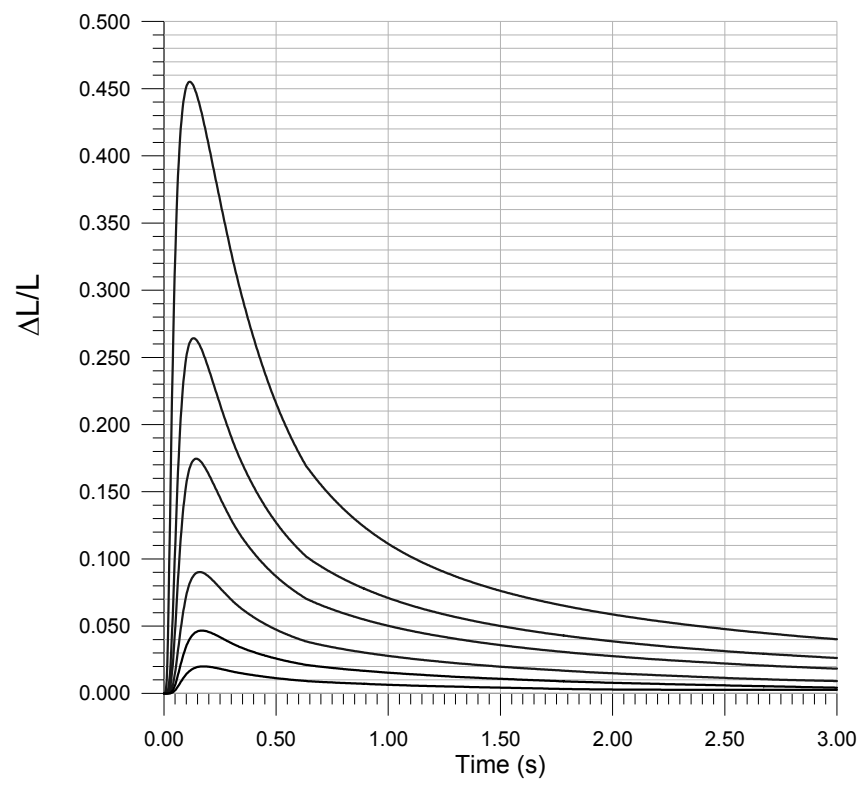

Fig. 4. Temporal distribution accordings to equation (1)

Table 1. The estimation of corrosion for particular defects

\begin{tabular}{|l|l|l|l|}
\hline $\begin{array}{l}\text { Effective } \\
\text { corrosion }\end{array}$ & $\begin{array}{l}\text { Estimation } \\
\text { time }\end{array}$ & $\begin{array}{l}\text { Estimation of } \\
\text { corrosion }\end{array}$ & Error \\
$\Delta L / L$ & Fo & $\Delta L / L$ & $\%$ \\
\hline 0,02 & 0,21 & 0,0199 & $-0,5$ \\
\hline 0,05 & 0,242 & 0,0466 & $-6,8$ \\
\hline 0,1 & 0,264 & 0,0902 & $-9,8$ \\
\hline 0,2 & 0,292 & 0,175 & $-12,5$ \\
\hline 0,3 & 0,31 & 0,264 & $-12,0$ \\
\hline 0,5 & 0,32 & 0,455 & $-9,0$ \\
\hline
\end{tabular}

The optimum time interval to estimate the defect contours (time interval during which the defect contours in the thermogram may be most accurately identified) is also the time interval for achieving the maximum $\Delta T$, where the reading of the diameter of each particular defect is done at the half of the amplitude. Figure 5 displays the line temperature distributions along the defects in the time instant Fo $=$ $0,28(\tau=0,15 \mathrm{~s})$. 

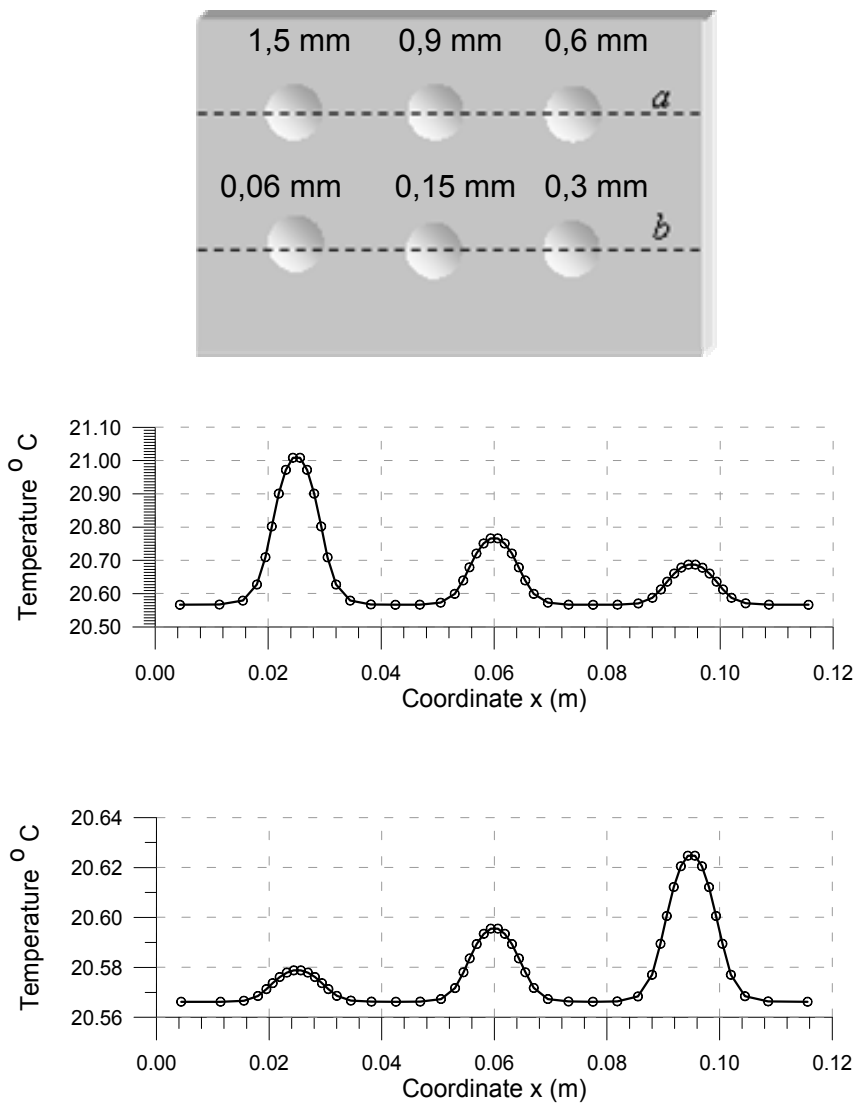

Fig. 5. a) Line temperature distribution for cross section a; b) Line temperature distribution for cross section $b$

The effective diameter of all the defects was $10 \mathrm{~mm}$. The measured defect diameters are given in Table 2.

Table 2. The measured defects diameters

\begin{tabular}{|l|l|l|l|l|l|l|}
\hline Effective corrosion $\Delta L / L$ & 0,02 & 0,05 & 0,1 & 0,2 & 0,3 & 0,5 \\
\hline Measured defect diameter $\mathrm{mm}$ & 10,16 & 10,16 & 9,83 & 9,83 & 9,83 & 9,5 \\
\hline
\end{tabular}

According to literature data, material loss defects above some $20 \%$ may be detected by thermography. The temporal temperature derivation according to equation (3) increases partially the defect visibility. In Figure 6, where the threedimensional surface temperature distribution in the time instant $0.1 \mathrm{~s}$ is shown, the smallest defect is not visible. On the other hand, the temporal derivation of temperature for $\tau_{1}=0,05 \mathrm{~s}$ and $\tau_{2} 0,1 \mathrm{~s}$ in Figure 7 displays all the defects. 


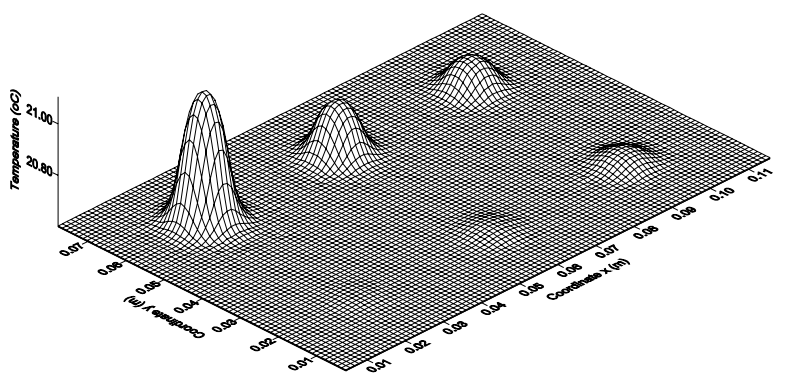

Fig. 6. Temperature distribution for the time instant $0,1 \mathrm{~s}$

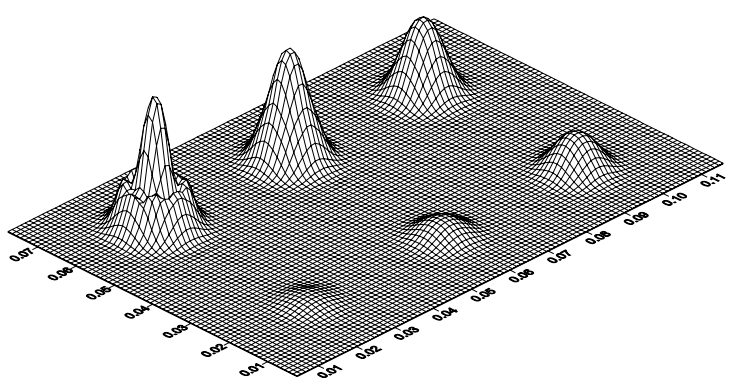

Fig. 7. Temporal temperature derivation $(0,05 s-0,1 s)$

\section{Conclusion}

The performed investigation yields the conclusion that both the thermographic and the numerical method may be successfully employed in thermal non-destructive testing. The numerical approach enables the simulation of the influence of particular parameters, thus enabling a more universal overview of the influencing values. By simulation of a process for a model with a defined number and distribution of defects it was established that the mutual influence of the defects is very important, which may be directly concluded from the experimental part of the investigation. The numerical simulation also indicates the situations when the three-dimensional diffusion may not be neglected. It was also shown that the selection of an optimal inspection time interval is essential for high quality evaluation of results.

It may be concluded that a high quality approach to thermal non-destructive testing necessarily requests a close collaboration of experiment and numerical analysis in order to detect and determine all the relevant defect parameters.

\section{REFERENCES}

[1] D.P.Almond, P.M.Patel, Photothermal Science and Technique, London, Chapman \& Hall, 1996. 
[2] S.V. Patankar, Numerical Heat Transfer and Fluid Flow, Hemisfere Publishing Corporation, Mc Grow-Hill Book Co, Washington, 1980.

[3] I.llić-Boras, S.Švaić, Numerical simulation of the defect in specimen, 4th International Conference of the Slovenian Society for NDT, Ljubljana, 1997.

[4] S.Marinetti, P.G.Bison, E.Grinzato, 3D Heat flux effects in the experimental evalustion of corrosion by IR thermography, QIRT'02, Quantitative InfraRed Thermography 6, Dubrovnik, Croatia, 2002.

[5] E.Grinzato, V.Vavilov, Corrosion evaluation by thermal image processing and 3D modelling, Rev.Gen.Therm. Paris, France, pp. 669-679,1998.

[6] I.Boras, S.Švaić, Determination of the Defect Parameters in Specimen by Means of Thermography and Numerical Methods, Proceeding of The International Society for Optical Engineering, San Antonio, Texas, USA, Vol. 3396, pp. 271-281, 1998. 